\title{
THE SIGNIFICANCE OF THE POLARIZATION OF SOLAR SHORT-WAVELENGTH X-RAYS
}

\author{
G. ELWERT \\ (Lehrstuhl für Theoretische Astrophysik, Universität Tübingen, Germany)
}

For the interpretation of the physical events occurring at the beginning of a flare it seems to be significant not only to observe the spectra of the short-wavelength $X$-ray radiation but also its polarization. Solar X-ray spectra below $10 \AA$ during flares observed by Bowen et al. (1964) show in the region of long wavelength a slow fall in their intensity and below $5 \AA$ approximately a rapid fall (Figure 1). This spectral variation cannot be understood on the basis of thermal radiation only. However, it is possible to explain the observed spectra by assuming a superposition of a thermal radiation as well as a non-thermal one. Indeed, one should assume that the fast electrons, during their acceleration, obtain a preferred direction and that only after-

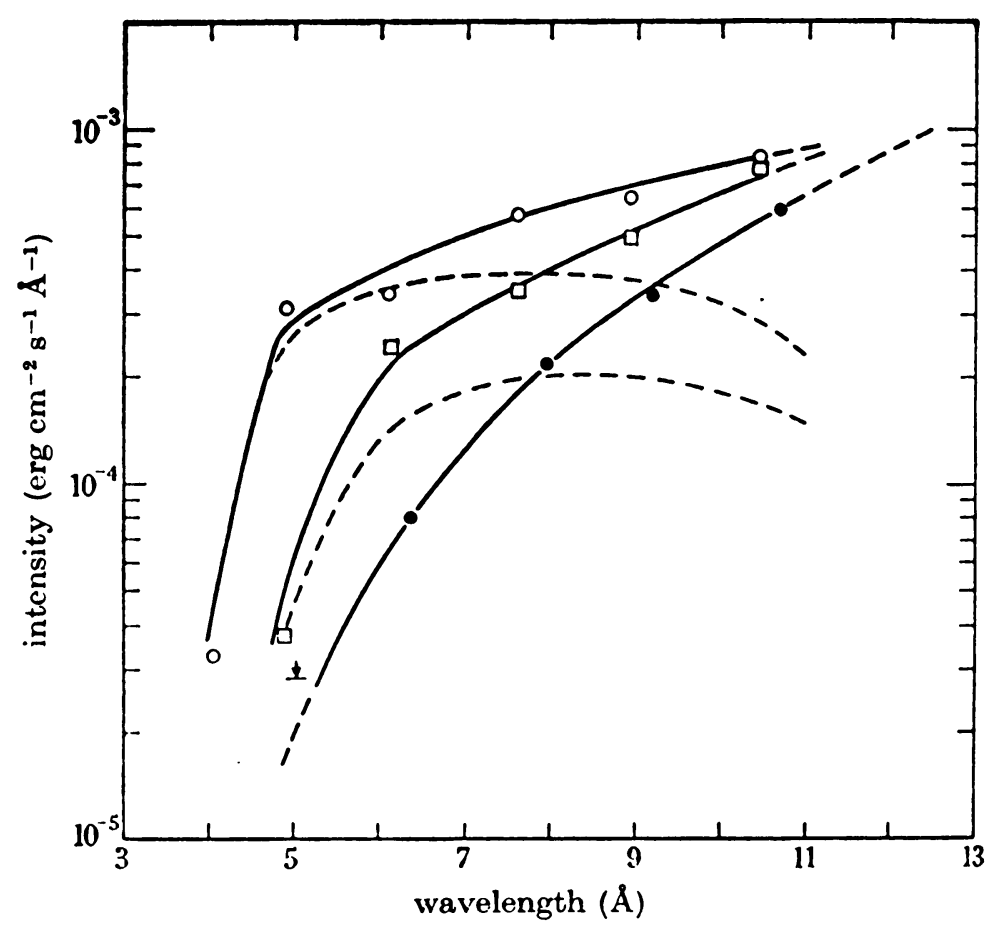

FIG. 1. Solar X-ray spectra as observed by Bowen et al. (1964).

Kiepenheuer (ed.), Structure and Development of Solar Active Regions, 444-448. C I.A.U. 
wards their velocity distribution becomes a Maxwellian one. The assumption of the existence of a non-Maxwellian distribution of electrons in the beginning of a flare is also supported by the fact that the rise in intensity by more than one order of magnitude takes place in a few minutes. This point has already been emphasised by Bowen et al.

Accordingly one is led to the interpretation that the non-thermal part is produced by electrons moving in a preferred direction. This interpretation then corresponds to the conjecture of De Jager and Kundu (1963), according to which electrons accelerated in a magnetically neutral zone of a flare and moving towards the photosphere generate $\mathrm{X}$-ray bremsstrahlung in the deeper atmosphere, while the outgoing electrons cause a type-III burst (Figure 2). The model proposed by Takakura and Kai (1966) would also provide electrons moving in a preferred direction. In this case the energetic electrons are trapped in a magnetic tube situated in the lower corona between bipolar sun spots (Figure 3). Now, the point is that for the fast electrons moving in a preferred

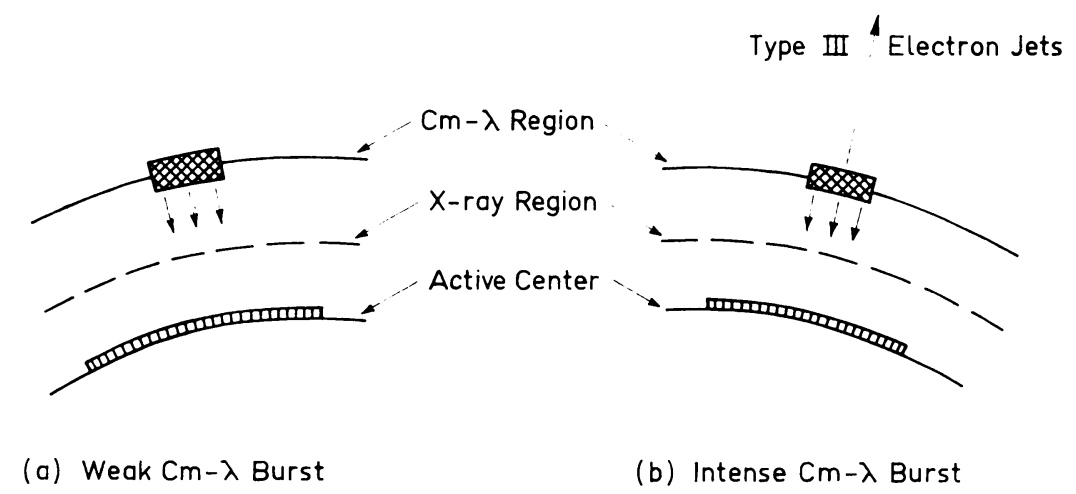

FIG. 2. Flare model according to De Jager and Kundu (1963).

$10^{5} \mathrm{~km}$

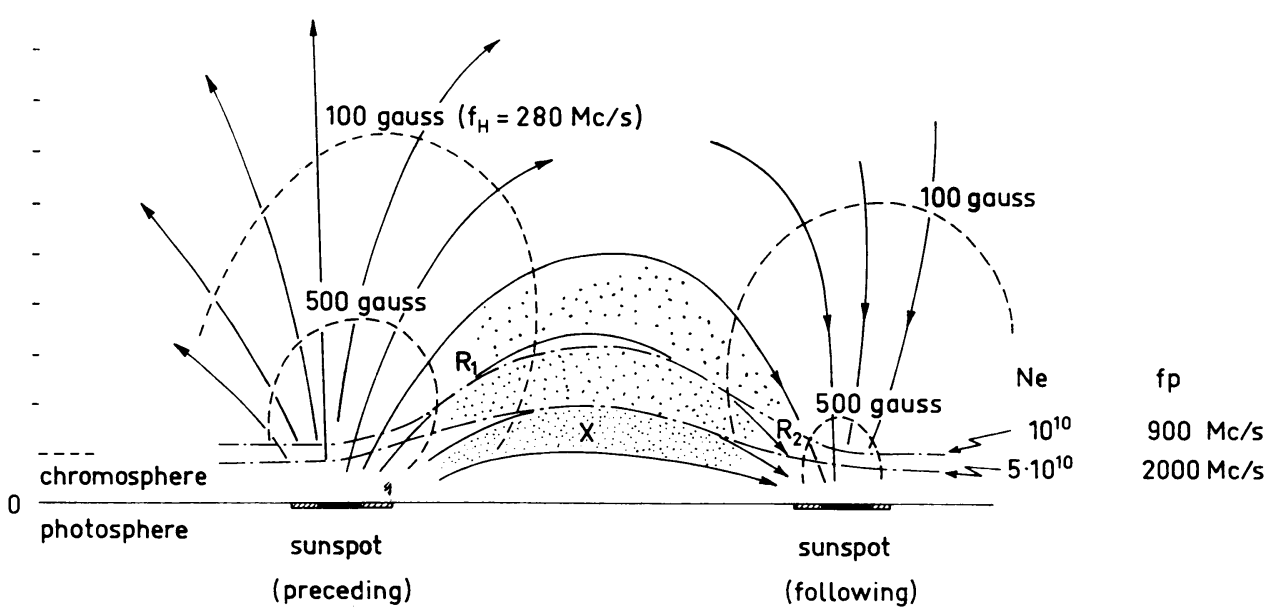

FIG. 3. Flare model according to Takakura and Kai (1966). 

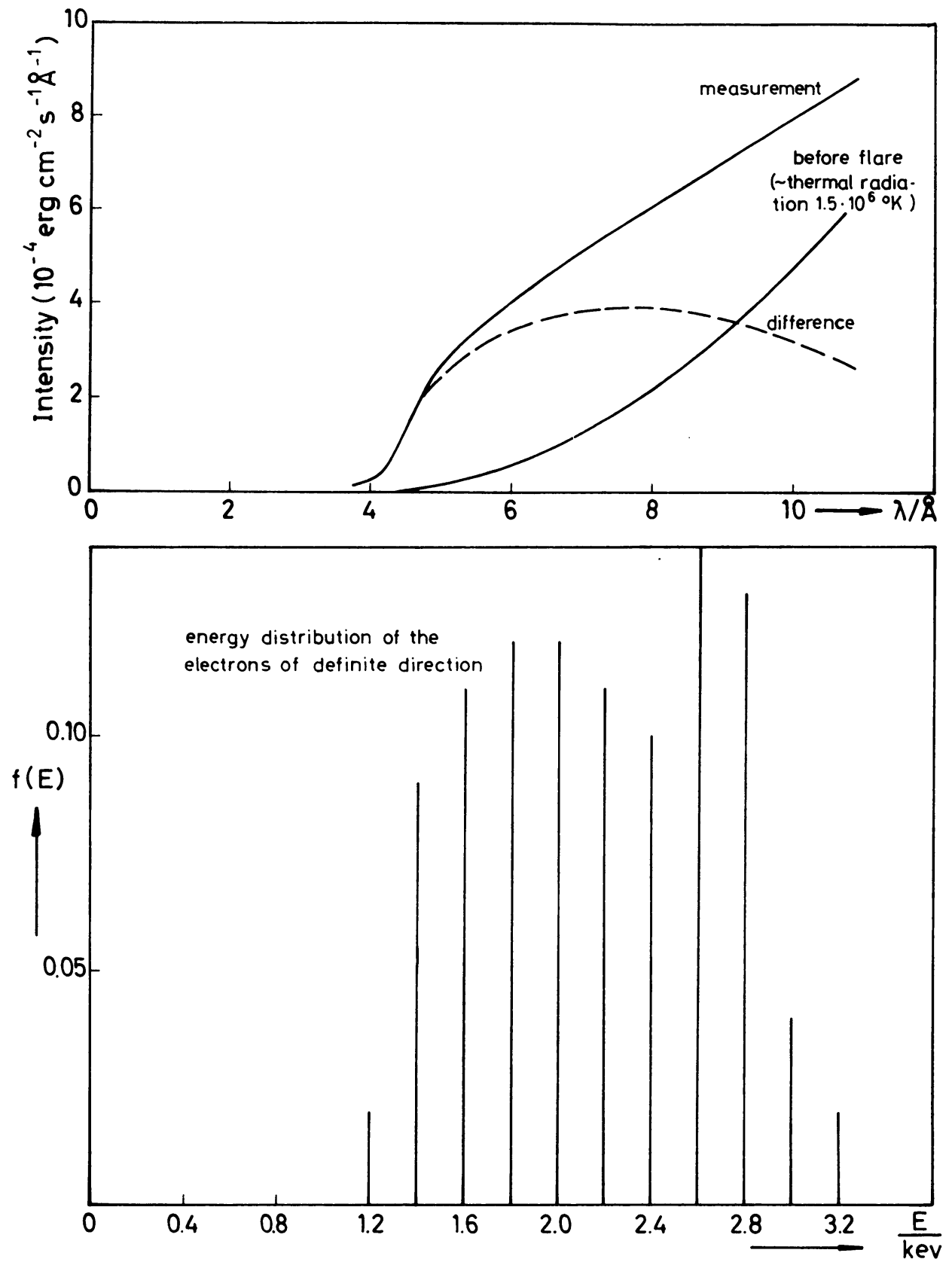

FIG. 4. X-ray spectra and distribution function of the electron energies $f(E)$. 
direction the X-ray bremsstrahlung should be appreciably polarized below $8 \AA$ approximately.

To get an idea of the polarization which can be expected, we discuss in the following the uppermost spectrum observed by Bowen $e t$ al. The intensity of the radiation before the flare corresponding to a temperature of $1.5 \times 10^{6}{ }^{\circ} \mathrm{K}$ is subtracted from the measured one to give the dashed curve. These curves are represented again in Figure 4. The distribution function $f(E)$ of the electron energy, which allows one to represent the difference curve as X-ray bremsstrahlung of directed electrons, was calculated neglecting a possible contribution of recombination radiation. This function $f(E)$ with a maximum of about $2 \mathrm{keV}$ can be approximated by a discrete energy spectrum, which is also shown in Figure 4.

The degree of polarization of the X-radiation was then determined as a function of the angle $\theta$ between the direction of observation and the direction of the electrons (Figure 5). This calculation, as well as the determination of $f(E)$, has been performed by one of my co-workers, Dr. Haug.

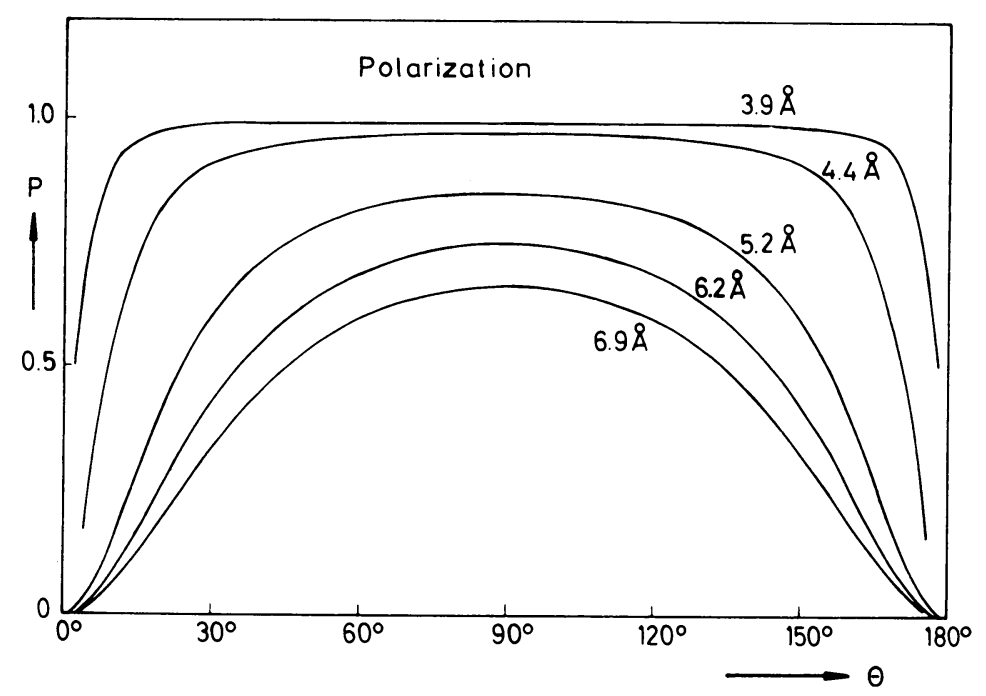

FIG. 5. Calculated $X$-ray polarization $P$.

The polarization $P$ attains its maximum value when observed at right angle to the direction of the electron beam $\left(\theta=90^{\circ}\right)$ i.e., in the case of a radial beam corresponding to the model of De Jager and Kundu for a flare at the limb of the Sun. On the other hand, the polarization goes to zero when observed opposite to the direction of the electron beam $\left(\theta=180^{\circ}\right)$, i.e., in the case of electrons moving inward radially for a flare occurring at the centre of the Sun's disk. For the model of Takakura and Kai, according to which the electrons move essentially parallel to the Sun's surface, the 
maximum value of $P$ would be obtained for a flare at the centre of the Sun's disk. We see from Figure 5 that the maximum values of polarization are nearly $60 \%$ at $7 \AA$ and practically $100 \%$ at $4 \AA$. Observed under $150^{\circ}$ with regard to the direction of the electron beam (in case of the model of De Jager and Kundu, i.e., for a flare occurring at a heliocentric angle of $30^{\circ}$ ), one obtains a polarization of nearly $30 \%$ at $7 \AA$ and practically $100 \%$ at $4 \AA$.

The spectrum used in our calculations was observed at the time of maximum intensity of X-ray flares. Thus, our calculations lead to the conclusion that it should be possible to establish during this time an appreciable degree of polarization of short-wave bremsstrahlung X-rays. By measuring the polarization, the models in which the fast electrons have a preferred direction could therefore be tested. The degree of polarization would increase with decreasing wavelength. If no polarization is found, each model in which the electrons have at all a preferred direction would then be ruled out. A proposal to perform these measurements has been accepted by the sun group of ESRO.

As Acton has reported (1968), the short-wavelength X-radiation below $3 \AA$ observed during undisturbed conditions can neither be explained as thermal radiation. Most probably, the non-thermal radiation is also generated as bremsstrahlung by electrons with a preferred direction. Consequently one can expect a substantial degree of polarization.

More details will be published elsewhere.

\section{References}

Acton, L.W. (1968) in the present volume, p. 432.

Bowen, P.J., Norman, K., Pounds, K. A., Sanford, P.W., Willmore, A.P. (1964) Proc. Roy. Soc., 281, 538.

De Jager, C., Kundu, M.R. (1963) in Space Research, III, p. 836.

Elwert, G., Haug, E. (1968) to be published.

Takakura, T., Kai, K. (1966) Publ. astr. Soc. Japan, 18, 57.

\section{DISCUSSION}

Öhman: Have you also developed some new schemes for measuring polarization?

Elwert: The polarization may be measured by standard technique, i.e. Thomson or Barkla effect; one measures the intensity of the scattered radiation as a function of the direction. Another possibility is to use the Borrmann effect. Borrmann has shown that X-rays incident under Braggs angle on a perfect crystal undergo no absorption and that the forward-diffracted beam is polarized orthogonal to the plane of scattering. 